\title{
A STOCHASTIC INVENTORY MODEL WITH STOCK DEPENDENT DEMAND ITEMS
}

\author{
LAKDERE BENKHEROUF \\ AMIN BOUMENIR \\ LAKHDAR AGGOUN \\ Sultan Qaboos University \\ Department of Mathematics and Statistics, PO Box 36 \\ Al-Khod 123, Sultanate of Oman
}

(Received April, 2000; Revised December, 2000)

\begin{abstract}
In this paper, we propose a new continuous time stochastic inventory model for stock dependent demand items. We then formulate the problem of finding the optimal replenishment schedule that minimizes the total expected discounted costs over an infinite horizon as a Quasi-Variational Inequality (QVI) problem. The QVI is shown to have a unique solution under some conditions.

Key words: Inventory Control, Impulse Control, Quasi-Variational Inequality.
\end{abstract}

AMS subject classifications: $90 \mathrm{~B} 05,60 \mathrm{~J} 60,49 \mathrm{~N} 25$.

\section{Introduction}

This paper discusses a single item continuous time stochastic inventory model for stock dependent demand terms. The discussion is motivated by the well known principle in the marketing literature that demand for certain items depends largely on the quantity displayed on the shelf (see for example, Corstjens and Doyle [2] and Schary and Becker [1]). There are some simple EOQ deterministic models such as Datta and Pal [3], Coswami and Chaudhuri [4] but no attempt has been made to incorporate this principle in continuous time stochastic inventory model due to the technical complication that arises from the inclusion of the stock dependent demand items.

To formulate the problem, let $x(t)$ denote the level of stock at time $t$. We assume that the cost structure of the model is the following:

(i) The discount factor is $\alpha$, with $\alpha>0$.

(ii) The holding cost is

$$
f(x)=\left\{\begin{array}{ccc}
-p x, & \text { for } x \leq 0 & \text { (shortage cost) } \\
q x, & \text { for } x>0 & \text { (holding cost) }
\end{array}\right.
$$


with $p>0$ and $q>0$.

(iii) The setup cost is $k$, with $k>0$.

(iv) A cost per unit of item is $c$, with $c>0$.

A replenishment policy consists of a sequence $\left(t_{i}, Q_{i}\right), i=1, \ldots$, where $t_{i}$ is the $i$ th time of ordering and $Q_{i}$ is the quantity ordered at time $t_{i}$, where $t_{1}<t_{2}<\ldots$ Let

and set

$$
\begin{gathered}
V_{n}=\left\{\left(t_{i}, Q_{i}\right)\right\}_{i=1, \ldots, n}, \\
\mathscr{F}_{n} \sigma\left\{x(s) ; s \leq t_{n}\right\},
\end{gathered}
$$

$$
V_{\infty}=\lim _{n \rightarrow \infty} V_{n}=V
$$

Assume that the variation in inventory is governed by the following stochastic differential equation

$$
d x_{t}=-\left(g+a x(t)^{\beta} I(x(t)>0)\right) d t-\sigma d w_{t}+\sum_{i \geq 0} Q_{i} \delta\left(t-t_{i}\right),
$$

where $I(A)$ is the indicator function of set $A, \delta$ is the Dirac function, $g>0, \sigma>0$, $a>0,0<\beta<1$, and $\left\{w_{t}\right\}$ is the standard Brownian motion. Note from (1) that when $\sigma=0$, then $\left(g+a x(t)^{\beta}\right)$ can be interpreted as the demand rate when $x(t)>0$. Also note from (1) that it is implicit in the model that when $x(t)<0$, shortages have no effect on the demand.

If $a=0$ then the above model reduces to the model found in Sulem [8]. However, our treatment is different from that of Sulem in a number of ways resulting in a more general approach.

Assume that $V_{n}$ is $\mathscr{F}_{n}$-measurable. Then, the optimal replenishment schedule reduces to the problem of finding the sequence $V^{*}$ that solves

$$
y(x)=\inf _{V}\left(E_{x}\left[\int_{0}^{\infty} f(x(t)) e^{-\alpha t} d t+\sum_{i \geq 0}\left(k+c Q_{i}\right) e^{-\alpha t_{i}}\right]\right),
$$

where the expectation is taken over all possible realizations of the process $x(t)$ under Policy V.

In the next section, we formulate the problem addressed in (2) as a Quasi-Variational Inequality (QVI) problem and show that under some conditions on the discount factor, the unit cost and the holding cost, a unique solution to the QVI exists. We conclude with some remarks on the problem of finding a replenishment schedule that minimizes the total cost per unit of time.

\section{The Quasi-Variational Problem and Optimal $(s, S)$ Policy}

In this section, we formulate the problem addressed in (2) as a Quasi-Variational Inequality (QVI) problem and show that the optimal impulse control policy is an $(s, S)$ policy, where $s$ and $S$ are determined uniquely under certain technicality conditions (see Theorem 1 below).

Fix $t$ and let $\tau$ be a short interval of time. We then have two cases:

(i) If $x(t)>0$ and no order is made in the interval $[t, t+\tau)$, then (1) and (2) 
imply that

$$
y(x) \leq E\left[\int_{t}^{t+\tau} f(x(t)) e^{-\alpha(s-t)} d s+y(t+\tau) e^{-\alpha \tau}\right]
$$

Write

$$
x(t+\tau)=x(t)+\Delta x \tau
$$

and use the fact that for a standard Brownian motion $w(t), E[w(t)]=0$, and $E\left[w^{2}(t)\right]=t$. The Taylor expansion of the right side of (3) gives

$$
\begin{gathered}
y(x) \leq \tau f(x(t))+y(x(t))+E\left[\Delta x_{\tau}\right] y^{\prime}(x(t)) \\
+\frac{1}{2} E\left[\Delta x_{\tau}\right]^{2} y^{\prime \prime}(x(t))-\alpha \tau y(x(t))-\alpha \tau E\left(\Delta x_{\tau}\right) y^{\prime}(x(t)) \\
-\frac{1}{2} \alpha \tau E\left(\left(\Delta x_{\tau}\right)^{2}\right) y^{\prime \prime}(x(t))+O\left(\tau^{2}\right)
\end{gathered}
$$

which leads to

$$
0 \leq \tau f(x(t))-\left(g+a x(t)^{\beta}\right) \tau y^{\prime}(x(t))+\frac{1}{2} \sigma^{2} \tau y^{\prime \prime}(x(t))-\alpha \tau y(x(t))+O\left(\tau^{2}\right) .
$$

Dividing by $\tau$ and letting $\tau \rightarrow 0$, gives

$$
-\frac{1}{2} \sigma^{2} y^{\prime \prime}(x(t))+\left(g+a x(t)^{\beta}\right) y^{\prime}(x(t))+\alpha y(x) \leq f .
$$

(ii) If $x(t) \leq 0$ and no order is made in the interval $[t, t+\tau)$, then a similar argument to the one used in $(i)$ gives

$$
-\frac{1}{2} \sigma^{2} y^{\prime \prime}(x(t))+g y^{\prime}(x(t))+\alpha y(x(t)) \leq f .
$$

(iii) If an order of size $Q$ is placed at time $t$, then the inventory level jumps from $x(t)$ to $x(t)+Q$. In other words,

$$
y(x(t)) \leq k+\inf _{Q}[c Q+y(x(t)+Q)] .
$$

Let $A$ and $M$ be two operators defined by

$$
\begin{gathered}
A y(x)=\left\{\begin{array}{lc}
-\frac{1}{2} \sigma^{2} y^{\prime \prime}(x)+\left(g+a x^{\beta}\right) y^{\prime}(x)+\alpha y(x), & \text { if } x>0 \\
-\frac{1}{2} \sigma^{2} y^{\prime \prime}(x)+g y^{\prime}(x)+\alpha y(x), & \text { if } x \leq 0 .
\end{array}\right. \\
M y(x)=k+\inf _{Q}[c Q+y(x+Q)] .
\end{gathered}
$$

Then the optimal expected costs for the inventory model is given as a solution of the QVI problem 


$$
\left\{\begin{array}{c}
A y \leq f \\
y \leq M y \\
(A y-f)(y-M y)=0 .
\end{array}\right.
$$

For more details on QVI, see Bensoussan and Lions [1].

To find the solution of the QVI problem given by (6), we follow Sulem [8] and divide the inventory space into two regions

(i) the continuation region

$$
C=\{x \in \mathbb{R} ; y(x)<M y(x)\}=\{x \in \mathbb{R} ; x>s\},
$$

where no order is made and

$$
A y=f
$$

where $A$ is defined in (4).

(ii) The stopping region

$$
\bar{C}=\{x \in \mathbb{R} ; y(x)=M y(x)\}=\{x \in \mathbb{R} ; x \leq s\},
$$

where $M$ is given by (5), corresponds to the states where an order is made. In $\bar{C}$, we have

$$
\begin{aligned}
y(x) & =k+\inf _{Q}[c Q+y(x+Q)] \\
& =k+c(S-x)+y(S)
\end{aligned}
$$

The solution to the QVI given by problem (6) is continuous differentiable and continuity at the boundary point $s$ gives from (8) that

$$
y^{\prime}(s)=-c .
$$

The infimum in (7) is attained at $S$. Hence,

$$
y^{\prime}(S)=-c
$$

Also, $y$ is continuous at $s$, which leads to

$$
y(S)=y(s)-k-c(S-s)
$$

Also, we require that

$$
\lim _{x \rightarrow+\infty} \frac{y(x)}{f(x)}<\infty
$$

Note at this stage, $s$ must be $<0$, otherwise (8)-(11) will lead to $s=S$, which means that $k=0$, contradicting the assumption that $k>0$. The following is the main result of the paper.

Theorem 1: There exists a unique solution to the $Q V I$ problem given in (6) if and only if $(-p+\alpha c)<0$. 
The proof of Theorem 1 is lengthy and thus is done in stages. In the first stage, we are concerned with the asymptotic nature of $y(x)+c x$ as $x \rightarrow+\infty$ and of $y(s)+c s$ as $s \rightarrow-\infty$.

In the second stage, we prove a series of results that eventually lead with the asymptotic result to the proof of Theorem 1 .

Let

$$
L(x)=y(x)+c x .
$$

Then we have:

Theorem 2: If $(-p+\alpha c)<0$, then

(i) $\quad \lim _{x \rightarrow+\infty} L(x)=+\infty$;

(ii) $\lim _{s \rightarrow \infty} L(s)=+\infty$.

Proof: Let

be the solution of

$$
y(x):= \begin{cases}y_{+}(x), & \text { if } x>0 \\ y_{-}(x), & \text { if } x \leq 0\end{cases}
$$

$$
A y(x):=\left\{\begin{array}{cc}
-p x, & \text { if } x>0 \\
q x, & \text { if } x \leq 0
\end{array}\right.
$$

with $A$ given by (4). Note that from (4) we have for $x>0$,

$$
-\frac{1}{2} \sigma^{2} y^{\prime \prime}+\left(g+a x^{\beta}\right) y^{\prime}+\alpha y=q x .
$$

Rewriting (14) as

$$
-y^{\prime \prime}+2 \frac{g+a x^{\beta}}{\sigma^{2}} y^{\prime}+2 \frac{\alpha}{\sigma^{2}} y=2 \frac{q}{\sigma^{2}} x
$$

Now, let

and

$$
\begin{aligned}
& P(x)=\frac{2}{\sigma^{2}}\left(g+a x^{\beta}\right), \\
& \bar{\alpha}=2 \frac{\alpha}{\sigma^{2}}, \quad \bar{q}=2 \frac{q}{\sigma^{2}}
\end{aligned}
$$

$$
y(x)=z(x) \exp \left\{\frac{1}{2} \int_{0}^{x} P(t) d t\right\} .
$$

Then it can be shown, after some algebra, that

$$
\begin{gathered}
-z^{\prime \prime}(x)+z(x) Q(x)=\bar{q} x \exp \left\{-\frac{1}{2} \int_{0}^{x} P(t) d t\right\} \\
Q(x)=\frac{1}{4} P^{2}(x)-\frac{1}{2} P^{\prime}(x)+\bar{\alpha} \\
=\frac{1}{\sigma^{4}}\left(g+a x^{\beta}\right)^{2}-\frac{a \beta}{\sigma^{2}} x^{\beta-1}+\bar{\alpha} .
\end{gathered}
$$

Using WKB method (see Olver [6, Chap. 6, Th. 2.1]), the complementary solutions of 
(15) as $x \rightarrow+\infty$ are asymptotically,

$$
\begin{gathered}
z_{1}(x) \approx Q^{-\frac{1}{4}}(x) \exp \left\{\int_{0}^{x} \sqrt{Q(t)} d t\right\} \\
z_{2}(x) \approx Q^{-\frac{1}{4}}(x) \exp \left\{-\int_{0}^{x} \sqrt{Q(t)} d t\right\}
\end{gathered}
$$

from which we get

$$
\begin{aligned}
& y_{1}(x) \approx z_{1}(x) \exp \left\{\frac{1}{2} \int_{0}^{x} P(t) d t\right\} \approx Q^{-\frac{1}{4}}(x) \exp \left\{\int_{0}^{x}\left(\sqrt{Q(t)}+\frac{1}{2} P(t)\right) d t\right\} \\
& y_{2}(x) \approx z_{2}(x) \exp \left\{\frac{1}{2} \int_{0}^{x} P(t) d t\right\} \approx Q^{-\frac{1}{4}}(x) \exp \left\{\int_{0}^{x}\left(-\sqrt{Q(t)}+\frac{1}{2} P(t)\right) d t\right\} .
\end{aligned}
$$

Note as $x \rightarrow 0, \sqrt{1+x} \approx 1+\frac{1}{2} x$ and from (16)

$$
\begin{aligned}
\sqrt{Q(x)} & =\sqrt{\frac{1}{4} P^{2}(x)\left\{1-2 \frac{P^{\prime}(x)}{P^{2}(x)}+4 \frac{\bar{\alpha}}{P^{2}(x)}\right\}} \\
& \approx \frac{1}{2} P(x)\left\{1-\frac{P^{\prime}(x)}{P^{2}(x)}+2 \frac{\bar{\alpha}}{P^{2}(x)}\right\}
\end{aligned}
$$

The above implies

$$
\begin{aligned}
\sqrt{Q(x)}+ & \frac{1}{2} P(x) \approx P(x)-\frac{P^{\prime}(x)}{2 P(x)}+\frac{\bar{\alpha}}{P(x)} \\
& \approx P(x)-\frac{\beta}{x}+\frac{\alpha}{a x^{\beta}} .
\end{aligned}
$$

Also,

$$
-\sqrt{Q(x)}+\frac{1}{2} P(x) \approx \frac{\beta}{x}-\frac{\alpha}{a x^{\beta}} .
$$

Using (18), we have

$$
\begin{aligned}
& y_{2}(x) \approx Q^{-\frac{1}{4}}(x) \exp \left\{\int_{0}^{x}\left(-\sqrt{Q(t)}+\frac{1}{2} P(t)\right) d t\right\} \\
& \approx \frac{\sigma}{\sqrt{a}} x^{-\frac{\beta}{2}} \exp \left\{\int_{0}^{x}\left(\frac{\beta}{t}-\frac{\alpha}{a t^{\beta}}\right) d t\right\}
\end{aligned}
$$




$$
\begin{gathered}
\approx \frac{\sigma}{\sqrt{a}} x^{-\frac{\beta}{2}} \exp \left\{\beta \log x-\frac{\alpha x^{1-\beta}}{a(1-\beta)}+\text { constant }\right\} \\
\approx \frac{\sigma}{\sqrt{a}} x^{+\frac{\beta}{2}} \exp \left\{-\frac{\alpha x^{1-\beta}}{a(1-\beta)}\right\} \rightarrow 0 \text { as } x \rightarrow \infty
\end{gathered}
$$

Similarly, it can be shown using (17) that

$$
\begin{aligned}
y_{1}(x) & \approx x^{-\frac{\beta}{2}} \exp \left\{\frac{2 a x^{\beta+1}}{\sigma^{2}(\beta+1)}\right\}, \\
& \approx \exp \left\{\frac{2 a x^{\beta+1}}{\sigma^{2}(\beta+1)}\right\} .
\end{aligned}
$$

Note that asymptotically, the general solution has the form

$$
y(x) \approx c_{1} y_{1}(x)+c_{2} y_{2}(x)+\tilde{y}_{p}
$$

where $y_{1}(x)$ and $y_{2}(x)$ are given respectively by (19) and (20) and that $\tilde{y}_{p}$ is the asymptotic particular solution to be found later.

Now, the growth condition (12) implies that $c_{1}=0$, which means that in order to show $(i)$, we only need to check that $\widetilde{y}_{p}$ is well behaved. To this end, we only need to look for a formal solution $\tilde{y}_{p} \approx \sum x^{\tau} \sum_{n} \geq 0 \frac{a_{n}}{x^{n}}$, which is an asymptotic series (see Olver [6]). Keep in mind that an asymptotic series may not converge.

There are several ways of finding the coefficients $a_{n}$ but here we use an iterative method. Rewrite (14) as

$$
y(x)=\frac{q}{\alpha} x+\frac{\sigma^{2}}{2 \alpha} y^{\prime \prime}-\frac{g+a x^{\beta}}{\alpha} y^{\prime}
$$

Define

$$
y_{n+1}(x)=\frac{\sigma^{2}}{2 \alpha} y_{n}^{\prime \prime}(x)-\frac{g+a x^{\beta}}{\alpha} y_{n}^{\prime}(x)
$$

where

$$
y_{0}=\frac{q}{\alpha} x
$$

The first iteration gives

$$
y_{1}(x)=-q \frac{g+a x^{\beta}}{\alpha^{2}} \approx \frac{a q x^{\beta}}{\alpha^{2}} .
$$

Then

$$
\begin{gathered}
y_{2}(x) \approx \frac{a q \sigma^{2}}{2 \alpha^{3}} \beta(\beta-1) x^{\beta-2}+\frac{a q\left(g+a x^{\beta}\right)}{\alpha^{3}} \beta x^{\beta-1} \\
\approx-\left(\frac{a}{\alpha}\right) x^{\beta}\left(\frac{\beta a q x^{\beta-1}}{\alpha^{2}}\right) .
\end{gathered}
$$


This suggests that $y_{n}(x)$ may be written as

$$
y_{n+1}(x) \approx(-1)^{n}\left(\frac{a}{\alpha}\right) x^{\beta} y_{n}^{\prime}(x)
$$

from which we deduce that

$$
y_{n} \approx \frac{q x}{\alpha}(-1)^{n} \frac{a^{n}}{\alpha^{n}} \beta(2 \beta-1) \ldots((n-1) \beta-(n-2)) x^{(\beta-1) n} .
$$

The series $\sum_{n>0} y_{n}$ is an asymptotic series since $y_{n+1}=o\left(y_{n}\right)$ (see Olver [6]). It follows that the particular solution is $\tilde{y}_{p}$ such that

$$
\tilde{y}_{p} \approx \frac{q}{\alpha} x
$$

Hence $(i)$ is true.

The argument used to show $(i)$ indicates that

$$
y_{+}(x)=\epsilon \phi_{1}(x)+y_{p+}(x)
$$

where $\phi_{1}(x)$ is the complementary solution and $y_{p+}(x)$ is a particular solution. Also, it can be shown that we have an explicit solution for $x<0$,

$$
y_{-}(x)=a e^{\lambda_{1}(x-s)}+b e^{\lambda_{2}(x-s)}+k_{1} x+k_{2}
$$

where

$$
\lambda_{1}=\frac{1}{\sigma^{2}}\left(g+\sqrt{g^{2}+2 \alpha \sigma^{2}}\right), \quad \lambda_{2}=\frac{1}{\sigma^{2}}\left(g-\sqrt{g^{2}+2 \alpha \sigma^{2}}\right),
$$

with $k_{1}=-\frac{p}{\alpha}, k_{2}=\frac{g p}{\alpha^{2}}, a$ and $b$ are to be determined.

Because the solution $y(x)$ of $A y=f$ is continuously differentiable, by matching $y_{-}(0)=y_{+}(0)$ and $y_{-}^{\prime}(0)=y_{+}^{\prime}(0)$, we get

$$
\begin{gathered}
a e^{-\lambda_{1} s}+b e^{-\lambda_{2} s}+k_{2}=\epsilon \phi_{1}(0)+y_{p+}(0) \\
a \lambda_{1} e^{-\lambda_{1} s}+b \lambda_{2} e^{-\lambda_{2} s}+k_{1}=\epsilon \phi_{1}^{\prime}(0)+y_{p+1}^{\prime}(0) .
\end{gathered}
$$

Using the condition $y_{-}^{\prime}(s)=-c$, after some algebra, leads to $y(s) \approx k_{1} s$ as $s \rightarrow-\infty$. It follows that

$$
L(s)=y(s)+c s \approx\left(c+k_{1}\right) s \rightarrow+\infty \text { as } s \rightarrow-\infty .
$$

This completes the proof.

Consider the differential equation

$$
A y=f
$$

with the conditions (9) and (12).

Lemma 3: If $(-p+\alpha c)<0$, then the solution $(s, S)$ satisfying $(9)-(12)$ exists. 
Proof: Write the solution of $(23)$ as $y(x, s)$. Let

$$
L(x, s)=y(x, s)+c x .
$$

Then it follows from (10), (11), and (13) that the problem of finding $(s, S)$ reduces to the problem of solving the system of nonlinear equations given by

$$
\begin{gathered}
L^{\prime}(S, s)=0, \\
L(s, s)=k+L(S, s) .
\end{gathered}
$$

It is clear from (9)-(11) that as $s \rightarrow 0, S \rightarrow 0$. Then

$$
L(s, s)<k+L(S, S), \text { as } s \rightarrow 0 .
$$

Also, we know by Theorem 2 that $L(s, s) \rightarrow+\infty$ as $s \rightarrow-\infty$ and $k+L(x, s) \rightarrow+\infty$ as $x \rightarrow+\infty$. Then there exists an $S\left(s^{*}\right) \in(-\infty,+\infty)$ such that $L^{\prime}\left(S^{*}(s), s\right)=0$ and $L\left(S^{*}(s), s\right)<\infty$, which implies that as $s \rightarrow-\infty, L(s, s)>k+L\left(S^{*}(s), s\right)$. The proof is then immediate.

Lemma 4: Assume that $(-p+\alpha c)<0$ and $(s, S)$ is the solution found from solving (15) with (9)-(12) satisfied. Then $L^{\prime \prime}(s) \geq 0$.

Proof: Assume that $L^{\prime \prime}(s)>0$. Note that $L(S)<L(s)$ implies from (7) that there exists some $x^{*} \in(s, S)$ such that $L^{\prime}\left(x^{*}\right)=0$. But $L^{\prime}(s)=0$. Hence, there exists some $Z \in\left(s, x^{*}\right)$ such that $L^{\prime \prime}(Z)=0$ and $L^{\prime}(Z)>0$. Thus, $Z$ is a local maximum of the function $L^{\prime}$.

Suppose first that $x^{*}<0$. Then (23) with $x<0$ implies that $L^{\prime \prime \prime}(Z)>0$, contradicting the assertion that $Z$ is a local maximum.

Now, if $x^{*}>0$, then (14) gives

$$
\alpha L\left(x^{*}\right)=(q+\alpha c) x^{*}+c\left(g+a x^{* \beta}\right)+\frac{1}{2} \sigma^{2} L^{\prime \prime}\left(x^{*}\right),
$$

with $L^{\prime \prime}\left(x^{*}\right) \leq 0$. But $L^{\prime}(S)=0$. Then there exists a turning point $Z^{*} \in\left(x^{*}, S\right)$ such that $L^{\prime}\left(Z^{*}\right)=0$ and $L^{\prime \prime}\left(Z^{*}\right)>0$, which implies

$$
\begin{aligned}
(q+\alpha c) x^{*}+c\left(g+a x^{* \beta}\right)+ & \frac{1}{2} \sigma^{2} L^{\prime \prime}\left(x^{*}\right)<(q+\alpha c) Z^{*}+c\left(g+a Z^{\beta_{*}}\right) \\
& +\frac{1}{2} \sigma^{2} L^{\prime \prime}\left(Z^{*}\right) .
\end{aligned}
$$

But this contradicts the fact that $L\left(x^{*}\right)>L\left(Z^{*}\right)$ which completes the proof.

Lemma 5: Under the assumptions of Lemma 3, we have

(i) $\quad L^{\prime}(x) \leq 0 ; s \leq S \leq S$,

(ii) $\quad L^{\prime}(x) \geq 0 ; \quad x \geq S$.

The proof of Lemma 5 is similar to that of Lemma 4 .

The following corollary follows immediately from Lemma 5.

Corollary 6: If $(-p+\alpha c)<0$, then if $s$ is known, $S$ is uniquely determined.

Theorem 7: Under the assumptions of Lemma 2, the $(s, S)$ policy is optimal for the QVI problem (6).

Proof: We need to check that the inequalities $y<M y$ for $x>s$, and $A y \leq f$ for $x \leq s$ are satisfied. 
Lemma 5 implies that the infimum of the expression $M y(x)$ in (5) is achieved at the point $\xi=S-x$ for $s \leq x \leq S$, and at $\xi=0$ for $x \geq S$. It follows that $M y(x)=k+c(S-x)+y(S)$, for $s \leq x \leq S$ and $M y(x)=k+\bar{y}(x)$, for $x \geq S$.

If $s \leq x \leq S$, we have

$$
y(x)-M y(x)=y(x)-k-c(S-x)-y(S) .
$$

It follows that

$$
(y(x)-M y(x))^{\prime}=y^{\prime}(x)-c=L^{\prime}(x) \leq 0 \text { by Lemma } 5 \text {. }
$$

Thus $y(x)-M y(x) \leq y(S)-M y(S)$. Also $y(x)-M y(x)=-k$ for $x \geq S$. Hence, $y(x)-M y(x)<0$ for $x>s$.

Next, we show that $A y \leq f$ for $x \geq s$. Note that for $x \leq s$, we have

$$
y(x)=k+c(S-x)+y(S)=y(s)+c(S-x)
$$

But $A y \leq f$ when $x \leq s$ which leads to

$$
-g c+\alpha y(s)+\alpha c s \leq(-p+\alpha c) x .
$$

Since $(-p+\alpha c)<0$ and $x \leq s \leq 0$, then it is enough to show that

$$
-g c+\alpha y(s)+\alpha c s \leq(-p+\alpha c) s
$$

or equivalently,

$$
-g c+\alpha y(s) \leq-p s
$$

Now (4) with Lemma 4 gives the desired result.

Lemma 8: If $(-p+\alpha c)<0$, then $(s, S)$ is the unique solution of the $Q V I$ problem given by $(6)$.

Proof: Assume that we have two solutions $\left(s_{1}, S\left(s_{1}\right)\right)$ and $\left(s_{2}, S\left(s_{2}\right)\right)$, with $s_{1}<s_{2}$. Then $L_{1}^{\prime}\left(s_{1}\right)=L^{\prime}\left(s_{2}\right)=0$ and $L^{\prime \prime}\left(s_{1}\right) \leq 0, L^{\prime \prime}\left(s_{2}\right) \leq 0$ by $(9)$ and Lemma 4 respectively. Then there exists $x^{*} \in\left(s_{1}, s_{2}\right)$ such that $L^{\prime \prime}\left(x^{*}\right)=0$ and $L^{\prime}\left(x^{*}\right)<0$. Further, we have $A y\left(x^{*}\right) \leq f\left(x^{*}\right)$, giving

$$
\alpha L\left(x^{*}\right)<(-p+\alpha c) x^{*}+g c .
$$

Also, we have $A y\left(x^{*}\right)=f\left(x^{*}\right)$, since $s_{1}<s_{2}$, giving

$$
-\frac{1}{2} \sigma^{2} L^{\prime \prime}\left(x^{*}\right)+g L^{\prime}\left(x^{*}\right)+\alpha L\left(x^{*}\right)=(-p+\alpha c) x^{*}+g c .
$$

However, the above is in contradiction with the assertion that $L^{\prime}\left(x^{*}\right)<0$ and $L^{\prime \prime}\left(x^{*}\right)=0$. This completes the proof.

Lemma 9: If $(-p+\alpha c) \geq 0$, then the QVI problem given by (6) admits no solution.

Proof: Assume that there is a solution $(s, S)$ to the QVI problem given by $(6)$. Then 


$$
A y \leq f, \text { for } x \leq s,
$$

or equivalently,

$$
L(x) \leq \frac{1}{\alpha}(-p+\alpha c) x+\frac{g c}{\alpha}
$$

If $(-p+\alpha c)>0$, then clearly $(25)$ is violated when $x \rightarrow-\infty$. Then in this case, $(s, S)$ cannot be a solution to the QVI problem.

Now assume that $(-p+\alpha c)=0$. Then $A y \leq f$ when $x \leq s$ which gives,

$$
L(S) \leq \frac{g c}{\alpha}
$$

which in turn leads by (11) and (13) to

$$
L(S) \leq \frac{g c}{\alpha}-k
$$

Using the fact that $A y(S)=f(S)$ and $L^{\prime}(S)=0$, we get

$$
-\frac{1}{2} \sigma^{2} L^{\prime \prime}(S)-\alpha k \geq\left\{\begin{array}{cc}
(-p+\alpha c) S & \text { if } S<0 \\
(q+\alpha c) S+a S^{\beta} c & \text { if } S>0 .
\end{array}\right.
$$

The left side of the above inequality is strictly negative while the right-hand side is strictly positive, which leads to a contradiction.

Theorem 1 follows from Theorem 2 and Lemmas 3-8.

Assume now that we are interested in impulse control policies, of the form $V=\left\{\left(t_{i}, Q_{i}\right)\right\}_{i=1}, \ldots$, where the $t_{i}$ are the ordering times and $Q_{i}$, the quantities ordered. The total cost per unit time is given by

$$
y_{V}(x)=\lim _{T \rightarrow \infty} \frac{E_{x}\left[\int_{0}^{T} f(x(t)) d t+\sum_{t_{i} \leq T}\left(k+c Q_{i}\right)\right]}{T} .
$$

where the dynamics of the process are given by (1) and the expectation is taken with respect to all realizations of the process. Then we say that $V^{*}$ is average cost optimal if

$$
y_{V^{*}}(x)=\inf _{V} y_{V}(x)
$$

Let $\mu=y_{V^{*}}(x)$. Then, it is known (see Lions and Perthame [5]), that the optimal cost $y$ in (2) behaves like $\left(\frac{\mu}{\alpha}+y_{0}\right)$ where $y_{0}$ satisfies some QVI problem that can be obtained from (6). Also, it is known that the optimal $(s, S)$ policy obtained from (6) converges to the optimal policy that minimizes the expected average future costs.

In this paper, we proposed a new continuous time stochastic inventory model for stock dependent demand items. We also formulated the problem of finding the optimal replenishment schedule that minimizes the total expected discounted costs over an infinite horizon, as a QVI. The QVI was shown to have a unique solution under some conditions. 


\section{Acknowledgement}

The authors would like to thank the anonymous referee for valuable comments on an earlier version of the paper.

\section{References}

[1] Bensoussan, A. and Lions, J.L., Impulse Control and Quasi-Variational Inequalities, Gauthier Villars, Paris 1984.

[2] Corstjens, M. and Doyle, P., A model for optimizing retail space allocation, Mgmt. Sci. 27 (1981), 822-833.

[3] Datta, T.K. and Pal, A.K., A note on an inventory model with inventory-level dependent demand rate, J. Opl. Res. Soc. 44 (1990), 971-978.

[4] Pal, S., Goswami, A. and Chaudhuri, K.S., A deterministic inventory model for deteriorating items with stock-dependent demand rate, Intern. J. of Prod. Econom. 32 (1993), 291-299.

[5] Lions, P.L. and Perthame, B., Quasi-variational inequalities and ergodic impulse control, SIAM J. Control. Optim. 24 (1986, 604-615.

[6] Olver, F.W., Asymptotics and Special Functions, Academic Press, New York 1974.

[7] Schary, P.B. and Becker, B.W., Distribution and final demand: The influence of availability, Mississippi Valley J. of Bus. and Econom. 8 (1972), 17-26.

[8] Sulem, A., A solvable one-dimensional model of a diffusion inventory system, Math. of Ops. Res. 11 (1986), 125-133. 


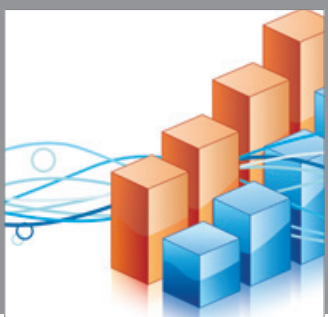

Advances in

Operations Research

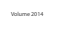

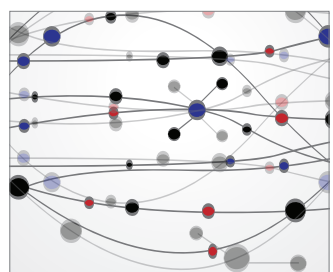

\section{The Scientific} World Journal
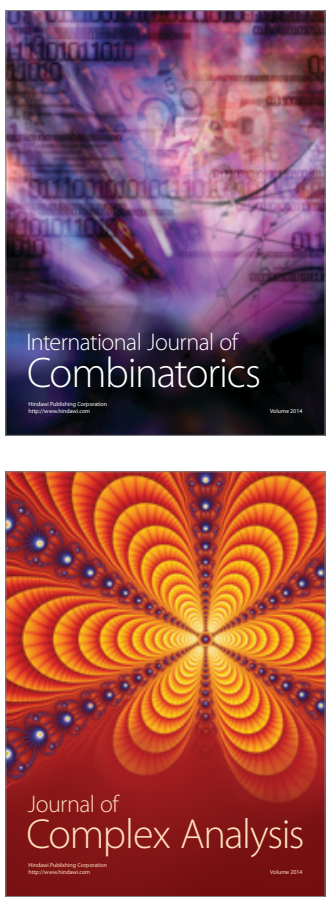

International Journal of

Mathematics and

Mathematical

Sciences
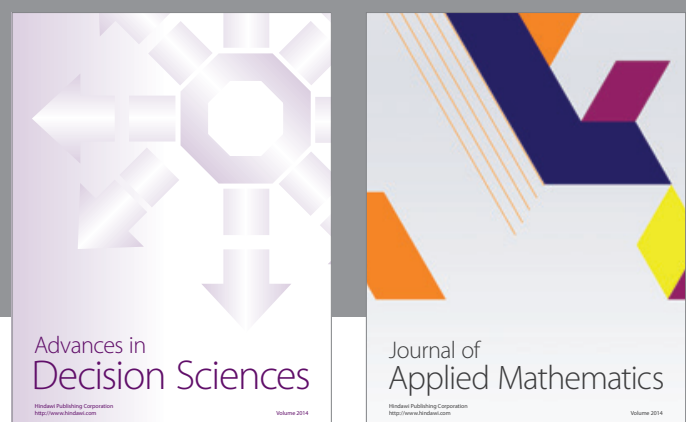

Journal of

Applied Mathematics
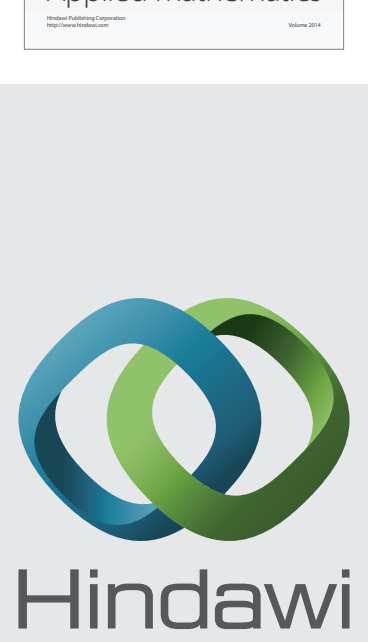

Submit your manuscripts at http://www.hindawi.com
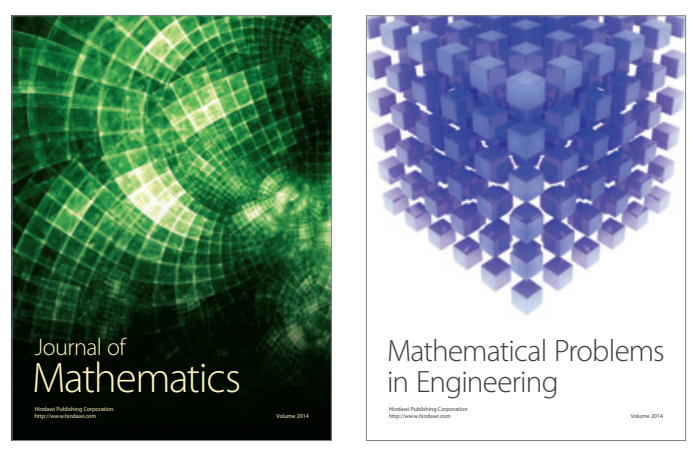

Mathematical Problems in Engineering
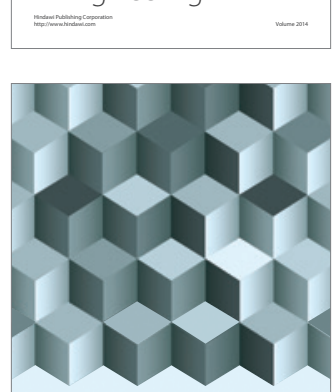

Journal of

Function Spaces
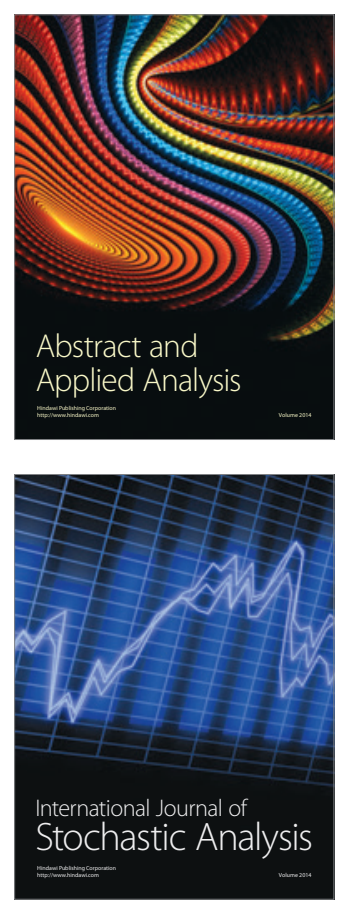

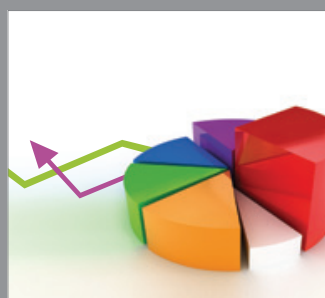

ournal of

Probability and Statistics

Promensencen
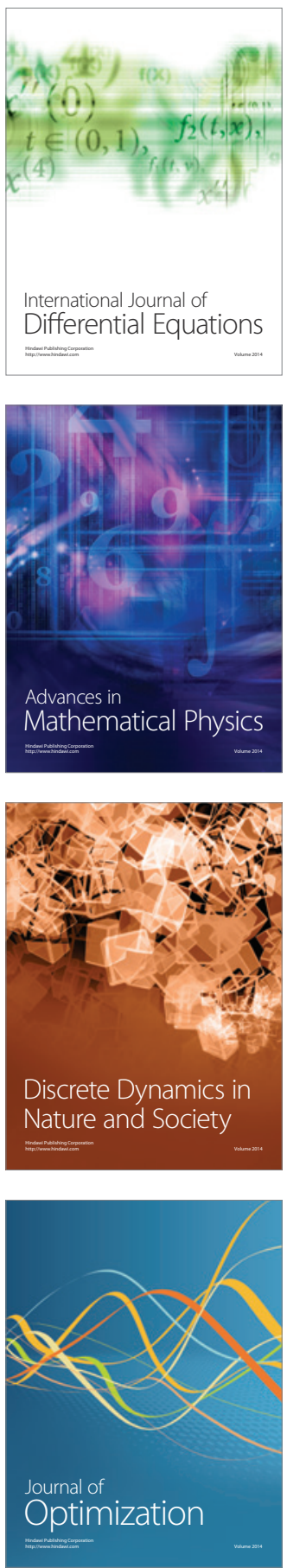